\title{
A possible X-ray jet from the starburst galaxy NGC 6217
}

\author{
W. Pietsch ${ }^{1}$ and H. Arp ${ }^{2}$ \\ 1 Max-Planck-Institut für extraterrestrische Physik, 85741 Garching, Germany \\ 2 Max-Planck-Institut für Astrophysik, 85741 Garching, Germany
}

Received 19 April 2001 / Accepted 6 July 2001

\begin{abstract}
Deep ROSAT PSPC and HRI observations of the nuclear starburst, UV flat spectrum spiral NGC 6217 reveal a jet-like X-ray filament extending out $2 ! 7(18.8 \mathrm{kpc})$ to the SW of the galaxy. Radio images of NGC 6217 show an extent in the same direction, giving further evidence for a one-sided X-ray jet interpretation of this feature. Its X-ray spectrum is harder than that of NGC 6217 and the luminosity in the $0.5-2.0 \mathrm{keV}$ band is $\sim 1.7 \times 10^{39} \mathrm{erg} \mathrm{s}^{-1}$. We compare our findings to parameters of other X-ray jets from other active galaxies. We also give positions of a total of $91 \mathrm{X}$-ray sources detected in the field to a limiting $0.5-2.0 \mathrm{keV}$ flux of $\sim 2.2 \times 10^{-15} \mathrm{erg} \mathrm{cm}^{-2} \mathrm{~s}^{-1}$ and propose optical and radio identifications. Some of these sources have been identified in the RIXOS program.
\end{abstract}

Key words. galaxies: individual: NGC 6217 - galaxies: jets - galaxies: quasars: general - galaxies: Seyfert radio continuum: galaxies - X-rays: galaxies

\section{Introduction}

NGC 6217 is a $B_{\mathrm{T}}=11.86$ mag barred spiral, RSBbc(s)II, as classified in the Revised-Shapley Ames Catalog of Bright Galaxies (Sandage \& Tammann 1981). It was first designated as abnormal in the Atlas of Peculiar Galaxies (Arp 1966a, b). In that classification it was No. 185, narrow filaments - between Nos. 149-152, jets and Nos. 209-215, material ejected from nuclei. The feature responsible for this classification was a narrow spiral arm-like filament coming off the end of the inner bar, much less wound up than the inner arms, which gave the impression of ejected material.

Subsequently the galaxy was described as "nuclear star burst, UV flat spectrum, infra-red bright" Seyfert 3 (Bonatto et al. 1998; Veron-Cetty \& Veron 1998). Observations of optical spectra dominated by stellar photoionization (Kennicutt 1992) and of extended radio emission at 1.46 and $4.9 \mathrm{GHz}$ (Hummel et al. 1984) and $\mathrm{CO}$ emission strongly peaked towards the center of the galaxy (Elfhag et al. 1996) support this classification. In a Hubble Space Telescope imaging survey of nearby active galactic nuclei, Malkan et al. (1998) classify the nuclear region (inner few arcsec) as CL, i.e. cluster, lumpy HiI region, knots, and DC, i.e. dust disk/dust lane passing close or through center.

NGC 6217 was detected in X-rays with the satellites Ginga and ROSAT (2-10 keV and $0.1-2.4 \mathrm{keV}$,

Send offprint requests to: W. Pietsch,

e-mail: wnp@mpe.mpg.de respectively) as discussed in a multi-wavelength catalog of Seyfert 2 galaxies observed in the $2-10 \mathrm{keV}$ energy band by Polletta et al. (1996). In addition, the galaxy is identified in one of the fields selected for the ROSAT International X-ray/Optical Survey (RIXOS, Mason et al. 2000) as source number 122-16. The narrow emission line galaxy (NELG) has been classified as a weak [O I] LINER with a power law photon index of the ROSAT spectrum of $1.7 \pm 0.1$ and time variability below $14 \%$ (Nicholson et al. 1997; Mittaz et al. 1999).

In this paper we will report on a careful analysis of all ROSAT observations of the NGC 6217 field aiming for the highest sensitivity well beyond the RIXOS threshold of $3 \times 10^{-14} \mathrm{erg} \mathrm{cm}^{-2} \mathrm{~s}^{-1}(0.5-2.0 \mathrm{keV})$ for point sources and diffuse emission. This led to the detection of a possible one-sided X-ray jet from NGC 6217 and a serendipitous PSPC survey at a Galactic viewing angle with moderate foreground absorption $\left(L_{\mathrm{II}}=111\right.$. $2, B_{\mathrm{II}}=33.5, N_{\mathrm{H}}=$ $4.1 \times 10^{20} \mathrm{~cm}^{-2}$; Dickey \& Lockman 1990) nearly as deep as dedicated ROSAT PSPC surveys (e.g. Hasinger et al. 1998). Throughout the paper we will assume a distance to NGC 6217 of $24.6 \mathrm{Mpc}$, i.e. $1^{\prime} \widehat{=} 7.2 \mathrm{kpc}$ (Condon et al. 1990).

\section{Observations and results}

During the ROSAT Wide Field Camera (WFC) "first light" observations the white-dwarf binary system RE J1629+780 was serendipitously detected as a bright source (Cooke et al. 1992). RE J1629+780 was then used 


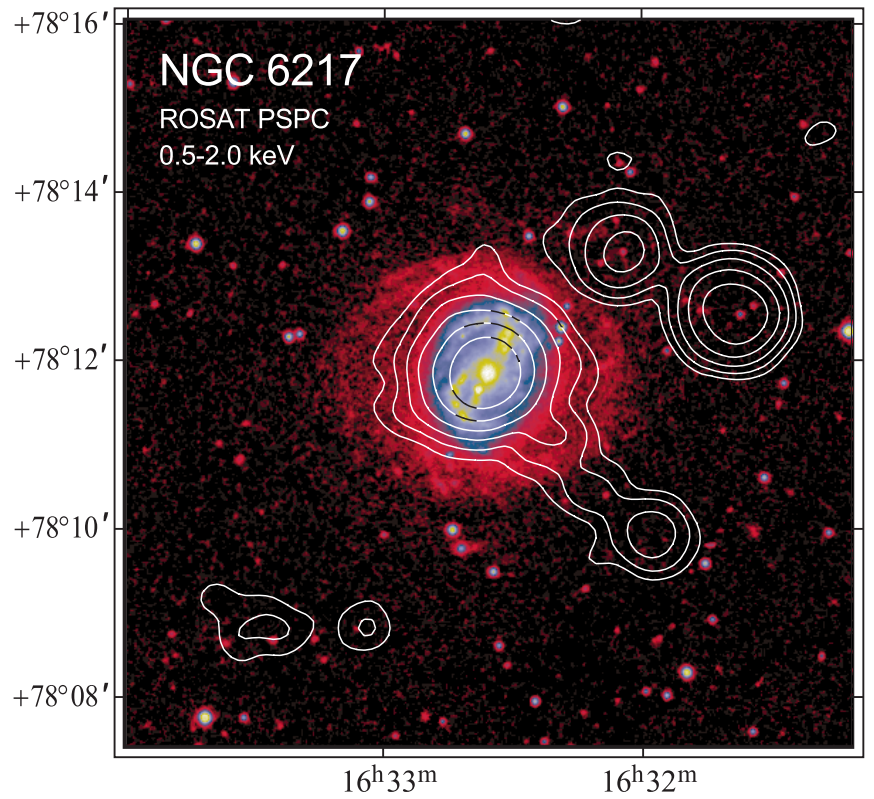

Fig. 1. Contour plot of the field around NGC 6217 for ROSAT PSPC hard band (0.5-2.0 keV) superimposed on an optical image extracted from the digitized sky survey II blue. The X-ray image has been smoothed according to the on-axis point spread function, X-ray contours are given in units of $\sigma\left(60 \times 10^{-6} \mathrm{ct} \mathrm{s}^{-1}\right.$ arcmin $\left.^{-2}\right)$ above the background $(170 \times$ $\left.10^{-6} \mathrm{ct} \mathrm{s}^{-1} \operatorname{arcmin}^{-2}\right)$. Contour levels are 5, 10, 20, 40, 80, and 160 units.

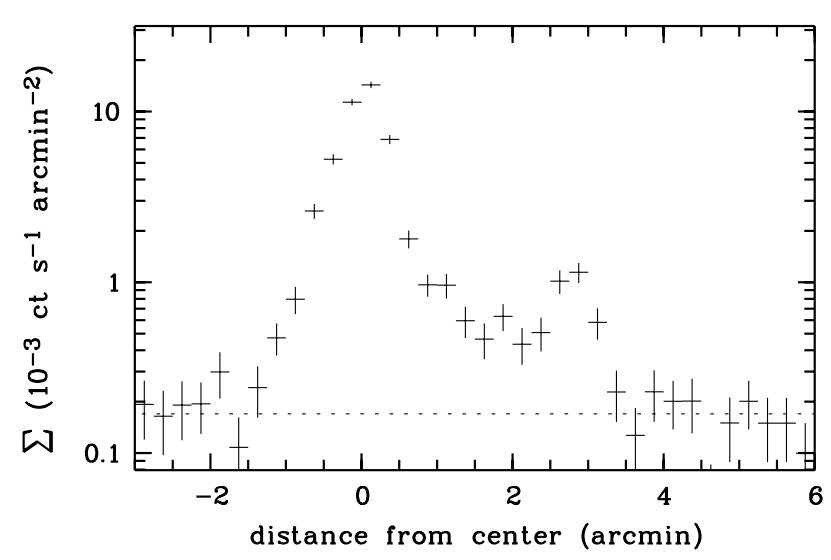

Fig. 2. Spatial distribution of surface brightness along position angle $226^{\circ}$ versus the distance from the NGC 6217 center. ROSAT PSPC hard $(0.5-2.0 \mathrm{keV})$ counts are integrated in boxes of $15^{\prime \prime} \times 90^{\prime \prime}$. The dotted line indicates the background surface brightness determined at distances of $\geq 1.5 \mathrm{NE}$ and $\geq 3.5$ SW of NGC 6217 .

throughout the entire ROSAT mission (Trümper 1982) as a WFC calibration source to monitor the gain and about a hundred PSPC and HRI (Pfeffermann et al. 1987) exposures were taken in parallel to the WFC observations. The total PSPC exposure time on RE J1629+780 is 129930 s made up of 31 pointings. Observation 142566p listed in the archive for the same pointing direction was rejected due to an offset from this nominal pointing

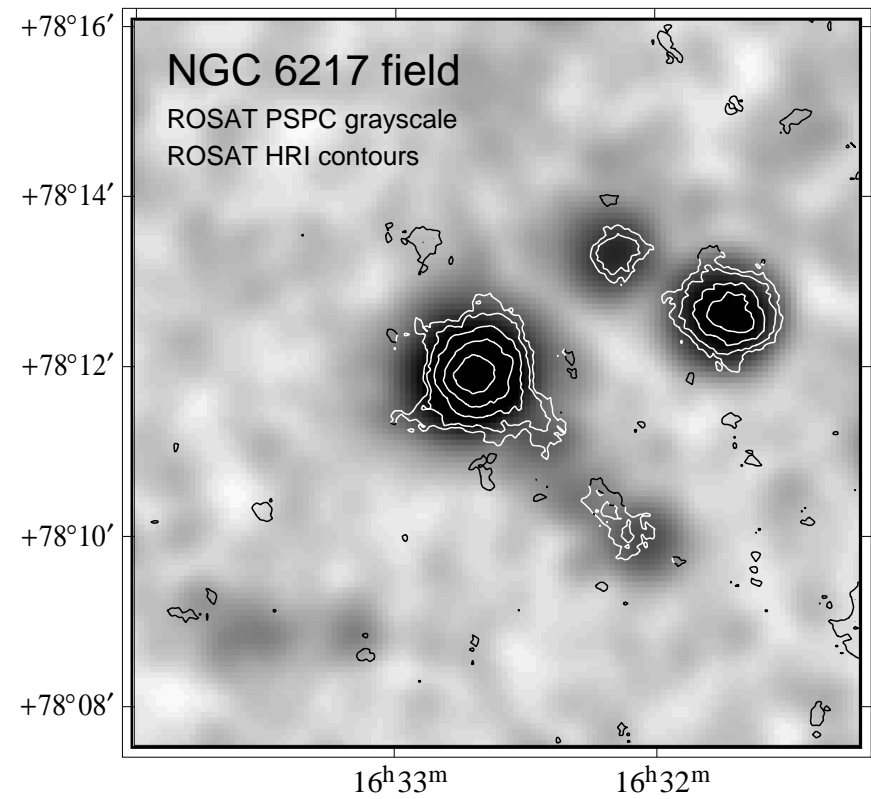

Fig. 3. Contour plot of the field around NGC 6217 from an adaptively smoothed ROSAT HRI image (see text) superimposed on a ROSAT PSPC hard band (0.5-2.0 keV) image. The PSPC image has been smoothed according to the onaxis point spread function. HRI X-ray contours are given in units of $\sigma\left(215 \times 10^{-6} \mathrm{ct} \mathrm{s}^{-1}\right.$ arcmin $\left.^{-2}\right)$ above the background $\left(2100 \times 10^{-6} \mathrm{ct} \mathrm{s}^{-1} \operatorname{arcmin}^{-2}\right)$. Contour levels are 3, 5, 10, 20, and 40 units.

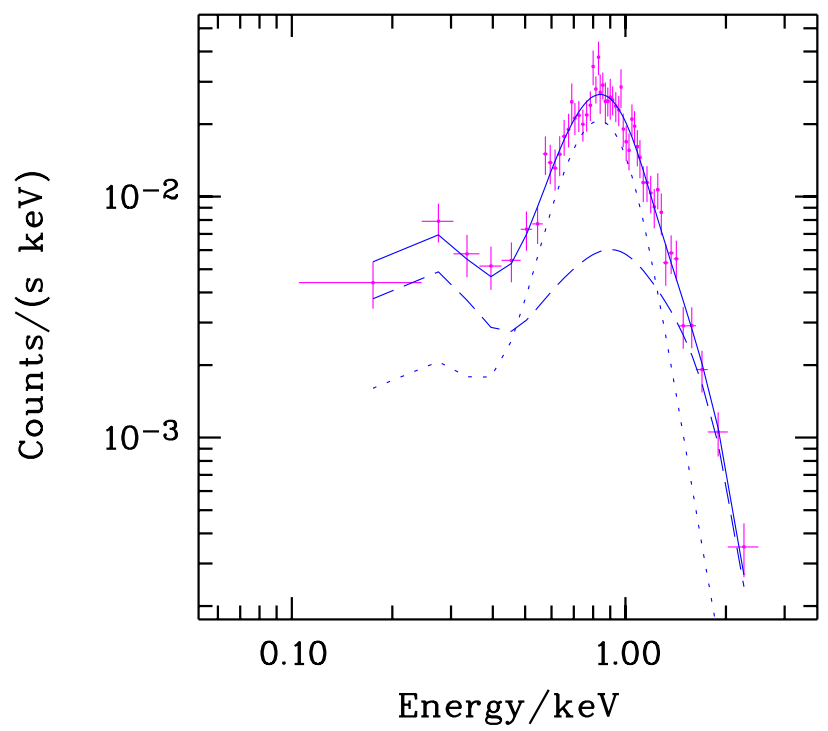

Fig. 4. Two component fit to the emission of NGC 6217. Count rate at the detector normalized to the energy, in counts $\mathrm{s}^{-1} \mathrm{~cm}^{-2}$, the crosses represent the measured count rates, the solid curve gives the best fit, the dotted curve represents the thin thermal, the dashed to the power law component (cf. Sect. 2).

direction of $\sim 6$ '4 mainly in right ascension. We also did not include two short observations in February 1998 to avoid detector degradation effects. The total HRI exposure time on RE J1629+780 is 247247 s made up of 67 pointings. NGC 6217 is offset from RE J1629+780 by $\sim 12^{\prime}$ 
to the NE and therefore during RE J1629+780 exposures well inside the ROSAT PSPC inner support ring and also inside the HRI field.

Source detection and position determination were performed over the inner $\sim 40^{\prime}$ field of view for each of the PSPC observations in the $0.5-2.0 \mathrm{keV}$ band (Zimmermann et al. 1998). The event sets had to be individually shifted by up to $10^{\prime \prime}$ in right ascension and $5^{\prime \prime}$ in declination with respect to an average position of the bright X-ray sources detected in each observation to correct residual boresight errors. The position-corrected data sets were then merged together. Though the PSPC observations were in general of rather low background, we removed times of high background in the $0.5-2.0 \mathrm{keV}$ band to increase the sensitivity for faint structures which reduced the useful exposure time by $\sim 10 \%$ to $117058 \mathrm{~s}$. The source detection procedures were re-run and source positions corrected using the RIXOS identifications (Mason et al. 2000) in the field reducing the systematic absolute position error to $0 . ! 8$ if one does not include an error due to the optically determined RIXOS positions. As Mason et al. (2000) state that the positional accuracy in the RIXOS catalog is better than $1^{\prime \prime}$, the residual systematic error will only give a significant contribution to the position error of the brightest sources and is not corrected for in the $90 \%$ uncertainty position error $r_{90}$. We also determined the likelihood of existence in the $0.5-2.0 \mathrm{keV}$ band $M L_{\text {exi }}$ and for the best position hardness ratios defined as $H R 1=(H-S) /(S+H)$ and $H R 2=(H 2-H 1) /(H 1+H 2)$ where $S, H, H 1$, and $H 2$ denote count rates in the $0.1-$ $0.4 \mathrm{keV}, 0.5-2.0 \mathrm{keV}, 0.5-0.9 \mathrm{keV}$ and $0.9-2.0 \mathrm{keV}$ bands, respectively. Taking into account the Galactic foreground absorption and folding a power law spectrum with a photon index of 2 through the instrument response, we determine a energy to count rates conversion factor in ct s $^{-1}$ in the $0.5-2.0 \mathrm{keV}$ band of 0.777 for a source with a flux of $1.0 \times 10^{-11} \mathrm{erg} \mathrm{cm}^{-2} \mathrm{~s}^{-1}$. Because the PSPC sensitivity is close to one in the hard band and variable foreground absorption mainly influences the soft band, this factor is almost insensitive to the assumed spectral shape and a wide range of $N_{\mathrm{H}}$.

The resulting list of 91 sources detected with $M L_{\text {exi }}>$ 10 in the $38.3 \times 38.3$ field centered on RE J1629+780 is only a by-product of the analysis of the field around NGC 6217. It reaches, however, much deeper than the published RIXOS catalog entries of the field and we therefore included it in the appendix (Table 2) together with an exposure-corrected hard band X-ray image (Fig. 3) with numbered boxes marking the catalog entries. Table 2 gives ROSAT name (Col. 1), source number (Col. 2), X-ray position (Cols. 3-4), $r_{90}$ (Col. 5), $M L_{\text {exi }}$ and count rate for the $0.5-2.0 \mathrm{keV}$ PSPC band (Cols. 6-7), hardness ratios HR1 and HR2 (Cols. 8-9), and some comments on possible identifications (Col. 10). The faintest sources in the catalog have a $0.5-2.0 \mathrm{keV}$ flux of $\sim 2.2 \times 10^{-15} \mathrm{erg} \mathrm{cm}^{-2} \mathrm{~s}^{-1}$.
The galaxy NGC 6217 is 13 '.3 from the center of the field. There seems to be an X-ray jet, emanating from the galaxy to about 2.7 projected at a position angle of $226^{\circ} \pm 2^{\circ}$ (Figs. 3 and 1 ). The X-ray surface brightness along the feature was integrated in $15^{\prime \prime} \times 90^{\prime \prime}$ boxes for the $0.5-2.0 \mathrm{keV}$ PSPC band (Fig. 2). From the surface brightness distribution we determine background-corrected count rates of $(162,8.5$, $9.6) \times 10^{-4}$ ct $\mathrm{s}^{-1}$ integrating the brightness at distances $-1.5-1.0,1.0-2 \cdot 25,2.25-3.5$, respectively, which can be attributed to NGC 6217 , an intermediate region, and the SW "knot". These count rates are in good agreement with the source detection results (see sources 73, 75,78 , and 80 of Table 2) and correspond to (151, 7.9, $9.0) \times 10^{38} \mathrm{erg} \mathrm{s}^{-1}$ at the distance of NGC 6217 . Source 80 represents NGC 6217, 73 the SW knot. Sources 75 and 78 are found in the bridge and at the "tongue" extending from the galaxy. Though we chose a small extraction radius for the source detection, specifically the results derived for source 78 and to a lesser amount for 75 may suffer from cross talk from sources 80 and 73 .

The count rate detected for NGC 6217 is within the errors the same as reported in the RIXOS survey (Mason et al. 2000) supporting the lack of time variability quoted by Nicholson et al. (1997). Mittaz et al. (1999) characterized the X-ray spectra of the extragalactic objects in the RIXOS survey based on power law fits on three broad-band X-ray colors assuming galactic absorption. This method only gives reasonable fits if the intrinsic spectrum really can be described by a power law. The number of photons of the RIXOS AGN identifications (source 39, 60,68 , and 88) as well as in NGC 6217 were sufficient to do fits with more than 10 and up to 52 data points in NGC 6217. For the AGN sample power law models gave acceptable fits with parameters coinciding within the errors with the RIXOS results. For NGC 6217 however, simple power law (and also thermal bremsstrahlung or thin thermal) models did not give acceptable fits (reduced $\chi^{2}>1.8$ ) even if the absorption column was not fixed to galactic. A combination of a thin thermal plasma of solar abundance and a power law with absorption for both components fixed to the galactic foreground resulted in an acceptable fit (reduced $\chi^{2}=0.66$ ). The temperature of the thermal component was $0.51 \pm 0.06 \mathrm{keV}$ and the power law photon index $1.8 \pm 0.3$ with absorption corrected luminosities in the $0.1-2.4 \mathrm{keV}$ band of $(1.1$ and 1.0$) \times 10^{40} \mathrm{erg} \mathrm{s}^{-1}$ (Fig. 4).

There are not enough counts in the jet-like feature to do a detailed spectral analysis. However, the hardness ratios in Table 2 of the sources detected therein indicate a somewhat harder spectrum than the power law spectrum with photon index of 1.7 of NGC 6217 (see Mittaz et al. 1999 and HR1 1.0, HR2 0.2 compared to 0.89 and 0.00 ). While the feature is clearly extended from NGC 6217 to the SW it cannot be resolved by the PSPC in the perpendicular direction. As Fig. 2 demonstrates, there is no indication of a similar feature on the opposite side of the galaxy. 


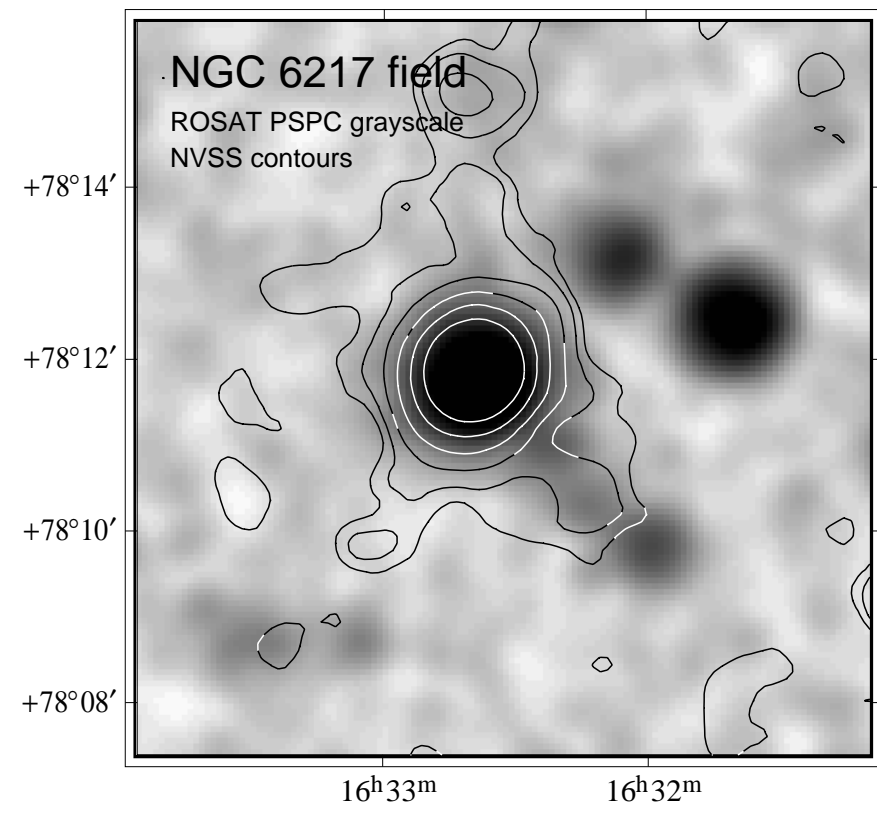

Fig. 5. Contour plot of the field around NGC 6217 from the NVSS Stokes I image (restoring beam width $45^{\prime \prime}$ FWHM, rms brightness fluctuations about $0.45 \mathrm{mJy} /$ beam) superimposed on the ROSAT PSPC hard band $(0.5-2.0 \mathrm{keV})$ image from Fig. 3. Radio contours are 1, 2, 4, 8, 16, and $32 \mathrm{mJy} /$ beam.

To analyze the HRI observations we merged the event sets, correcting the pointing directions with a time resolution of $40 \mathrm{~s}$ using the bright soft source RE J1629+780 as reference. Finally, we screened the data for times of high background which reduced the useful time of exposure by more than $27 \%$ to $179793 \mathrm{~s}$. Because the PSPC collects at least 2.5 times the photons and in addition has a lower background than the HRI, the faint emission to the SW of NGC 6217 is just marginally detected. Due to the relatively large off-axis position of NGC 6217 one also does not gain much due to the better intrinsic spatial resolution of the HRI as the point spread function is dominated by the X-ray telescope (Aschenbach 1988). However, the HRI results still can be used to confirm the PSPC result. To further reduce the background due to UV emission and cosmic rays, we used HRI raw channels 2-8. An HRI image (0.1-2.4 keV) was constructed with a bin size of 2.5 and adaptively smoothed by convolution with a circular top hat kernel using 100 counts. Iso-intensity contours of the resulting HRI image are superimposed on a PSPC $0.5-2.0 \mathrm{keV}$ gray-scale image in Fig. 3 and nicely confirm the brighter PSPC structures with slightly better resolution.

\section{Discussion}

Both in the PSPC and the HRI the main part (at least $\sim 80 \%$ ) of the X-ray emission of NGC 6217 is not resolved and appears as a point-like source with a jet-like feature emanating to the SW. The thin thermal component of the unabsorbed X-ray spectrum of NGC 6217 most likely arises in the nuclear starburst area of the galaxy, while the power law may reflect the unabsorbed jet component within a few $\mathrm{kpc}$ of the nucleus. The active nucleus most likely is heavily obscured and - similar to many other Seyfert 2 nuclei - not detected in the ROSAT band (see e.g. Moran et al. 2001). The radio flux density of NGC 6217 is close to that of the optical flux density and the galaxy therefore has to be classified as radio quiet (Kellermann et al. 1989).

Before discussing the X-ray feature to the SW of NGC 6217 as an X-ray jet we want to investigate the question as to whether the extent could be an accidental alignment of unrelated X-ray sources. Figures 1, A.1, and also Fig. 3 show that this could be possible. However, it would require at least three very closely spaced sources to be aligned rather exactly with the nucleus of the galaxy. This seems rather unlikely but certainly can not be excluded by the PSPC and HRI observations. Therefore we have to look for additional arguments from other wavelengths.

Radio observations provide additional evidence for jetlike emission from NGC 6217. There is not only arcsecscale extended emission from the nuclear area (Hummel et al. 1984), but also VLA radio maps at $1.49 \mathrm{GHz}$ (Condon 1987) and NRAO VLA Sky Survey (NVSS, Condon et al. 1998) reveal a complicated extended radio source on arcmin-scale with an extension coming out to $\sim 2^{\prime}$, exactly along the line of the X-ray extension (see Fig. 5) which might represent a radio jet. Unfortunately, the resolution and sensitivity of the observations is not sufficient to make a clear radio jet identification.

There is no optical correspondence at the position of the X-ray feature on either the POSS II plates (see Fig. 1 for an overlay of PSPC $0.5-2.0 \mathrm{keV}$ band contours on the digitized POSS II blue image) or the 200-inch telescope photograph. Also the Hubble Space Telescope imaging survey of nearby active galactic nuclei (Malkan et al. 1998) reveals no clear hint of a jet from the inner few arcsec around the nucleus. Since the interior of the galaxy is barred, it is difficult to judge the position of the minor axis. But using the outer isophotes of the Schmidt plates it is possible to estimate that the direction of the $\mathrm{X}$-ray feature is in the quadrant in which the minor axis lies (and also perpendicular to the bar). On the other hand, there are also no clear optical counterparts along the X-ray feature, as might be expected if it were an accidental alignment of unrelated sources. Such counterparts have been found for many of the other sources of similar X-ray brightness in the field (see Table 2).

For the further discussion we assume that the X-ray feature originates from the nuclear area of NGC 6217 . This then would point towards two possible explanations for the feature, a galactic superwind driven by a nuclear starburst or a one-sided jet originating from an active nucleus in NGC 6217.

Nuclear starbursts have been observed for several nearby galaxies leading to X-ray emission far out in the halos of galaxies (e.g. Dahlem et al. 1998). However, there the X-ray emission only stays collimated within the disk of 
Table 1. Parameters of X-ray jets.

\begin{tabular}{lrrrlll}
\hline Source & $\begin{array}{c}\text { Distance } \\
{[\mathrm{Mpc}]}\end{array}$ & \multicolumn{1}{c}{$\begin{array}{c}\text { Extent } \\
{\left[{ }^{\prime \prime}\right]}\end{array}$} & $\begin{array}{l}L_{\mathrm{X}} \\
{[\mathrm{kpc}]}\end{array}$ & $\begin{array}{c}\text { Comments } \\
{\left[\mathrm{erg} \mathrm{s}^{-1}\right]}\end{array}$ & & Ref. \\
\hline Cen A & 3.5 & 240 & 2.8 & $2.4 \times 10^{39}$ & $0.1-2.4 \mathrm{keV}$ band, radio, faint X-ray counter-jet & 1,2 \\
M87 & 17.0 & 11.5 & 1.0 & $1.9 \times 10^{40}$ & $2-10 \mathrm{keV}$ band, radio, optical, one-sided & 3 \\
NGC 6217 & 24.6 & 160 & 18.8 & $1.7 \times 10^{39}$ & $0.5-2.0 \mathrm{keV}$ band, radio, one-sided & this work \\
Pictor A & 151 & 115 & 85 & $6.8 \times 10^{40}$ & $2-10 \mathrm{keV}$ band, radio, one-sided, hot spot & 4 \\
3C273 & 550 & 22 & 60 & $4.0 \times 10^{43}$ & $0.5-8 \mathrm{keV}$ band, radio, optical, one-sided & 5 \\
PKS 0637-752 & 1200 & 12 & 70 & $1.5 \times 10^{44}$ & $2-10 \mathrm{keV}$ band, radio, optical, one-sided & 6 \\
\hline
\end{tabular}

References and notes:

(1) ROSAT HRI, Döbereiner et al. (1996); (2) Chandra, Kraft et al. (2000); (3) XMM-Newton, Böhringer et al. (2001);

(4) Chandra, $L_{\mathrm{X}}$ of hot spot $8.4 \times 10^{41} \mathrm{erg} \mathrm{s}^{-1}$, Wilson et al. (2001); (5) Chandra, Marshall et al. (2001); (6) Chandra,

Schwartz et al. (2000).

the galaxies and fills large parts of the galaxy halos, as e.g. the plume and outer halo emission in NGC 253 (Pietsch et al. 2000) or the nuclear superbubble and halo emission of NGC 3079 (Pietsch et al. 1998) show. Also the spectrum of the emission in the halo in general is significantly softer than the emission from the galaxy nuclear area and disk. No knot-like radio emission has been reported from X-ray halos fed by starbursts. Therefore a galactic superwind is a rather unlikely explanation.

This leaves the interpretation as a one-sided X-ray jet. The X-ray morphology would indicate that at least three knots with distances of $\sim 1^{\prime}, 1$ '.8, and 2 .7 from the nucleus contribute to the emission. The knot at 1!8 also shows up as a radio knot (see Fig. 5).

$\mathrm{X}$-ray jets with and without knot-like substructure have been reported for several AGN. The most famous X-ray jets are those in Cen A, M 87, Pictor A, 3C273, and PKS 0637-752, all radio-loud objects. However there is also the precessing jet in the radio-quiet galaxy NGC 4258 (Pietsch et al. 1994; Cecil et al. 2000) that also manifests itself in X-rays in the anomalous arm structure seen in projection onto the disk in the halo of this LINER/Seyfert galaxy. X-ray images from Chandra are now resolving the spatial structure along quasar jets, producing remarkable images. In Table 1 we have put together properties of these jets collected mainly from the new Chandra observations to compare with the NGC 6217 jet properties. To allow easier comparison, we have converted all numbers assuming $q_{0}=0.5$ and $H_{0}=70 \mathrm{~km} \mathrm{~s}^{-1} \mathrm{Mpc}^{-1}$. We give distance, apparent projected X-ray dimension in arcsec and kpc, respectively, the X-ray luminosity and we comment on the energy band used for the luminosity determination, and on radio and optical identification of the jets and give references. These X-ray jets are all strongly one-sided, as would be the proposed jet in NGC 6217 and are all also observed as radio jets or as colinear radio lobes. They also show hard X-ray spectra as proposed from the hardness ratios for the NGC 6217 jet.

According to Table 1 the NGC 6217 jet would be one of the longest in apparent dimensions and would seem to merit higher resolution from Chandra or greater wavelength coverage investigations from XMM-Newton in order to learn more about the characteristics of energetic ejections. Its measured luminosity is only moderate. However, jet emission may in part be hidden within the flux attributed to NGC 6217 itself becaused of the limited resolution of the ROSAT PSPC, as already is indicated by the slightly better resolution of the HRI which detects jet emission closer to the galaxy nucleus (cf. Fig. 3). The missing optical evidence for a jet in NGC 6217 is no strong argument. Also in Cen A there is no particular optical object associated with the X-ray jet although there are some outer gaseous filaments which lie along the projected track and in Pictor A there is no optical jet visible even in large telescope images. While this, together with the faintness in $\mathrm{X}$-ray and radio, may explain that we do not see hints for jet emission in the optical, deeper, large telescope imaging and radio observations would strongly be needed in addition to better X-ray data to give more strength to the proposed jet interpretation.

Acknowledgements. The X-ray data used in this work were all obtained from the ROSAT Data Archive at the Max-PlanckInstitut für extraterrestrische Physik (MPE) at Garching. This research has made use of the NASA/IPAC Extragalactic Database (NED) which is operated by the Jet Propulsion Laboratory, California Institute of Technology, under contract with the National Aeronautics and Space Administration. To overlay the X-ray data we used a blue digitized sky survey II image. The compressed files of the "Palomar ObservatorySpace Telescope Science Institute Digital Sky Survey" of the northern sky, based on scans of the Second Palomar Sky Survey are copyright (c) 1993-1997 by the California Institute of Technology. All material not subject to the above copyright provision is copyright (c) 1997 by the Association of Universities for Research in Astronomy, Inc. Produced under Contract No. NAS5-2555 with the National Aeronautics and Space Administration. The ROSAT project is supported by the German Bundesministerium für Bildung, Wissenschaft, Forschung und Technologie (BMBF/DLR) and by the MaxPlanck-Gesellschaft (MPG). 
Table 2. X-ray sources detected with the ROSAT PSPC in the $0.5-2 \mathrm{keV}$ band in a field of $38^{\prime} \cdot 3 \times 38^{\prime} \cdot 3$ centered on RE J1629+780.

\begin{tabular}{|c|c|c|c|c|c|c|c|c|c|c|c|}
\hline 1 & 2 & 3 & & 4 & 5 & 6 & 7 & 8 & 9 & 10 & 11 \\
\hline Source name & No & RA (J2000. & & Dec & $r_{90}$ & $M L_{\mathrm{exi}}$ & Count rate & $H R 1$ & $H R 2$ & Identifications & $\log \left(\frac{f_{\mathrm{x}}}{f_{\mathrm{V}}}\right)$ \\
\hline & & {$[\mathrm{h} \mathrm{m} \mathrm{s}]$} & & $\left.{ }^{\prime}{ }^{\prime \prime}\right]$ & {$\left[{ }^{\prime \prime}\right]$} & & {$\left[\mathrm{ct} \mathrm{s}^{-1}\right]$} & & & & \\
\hline RX J162300.6+781315 & 1 & 162300.64 & 781 & 1316.0 & 19.5 & 20.8 & $5.08 \mathrm{e}-04 \pm 1.0 \mathrm{e}-04$ & $0.31 \pm 0.25$ & $-0.31 \pm 0.19$ & U56154(17.0,0.5,23.9) & -2.2 \\
\hline RX J162327.7+775453 & 2 & 162327.78 & 775 & 5453.2 & 6.6 & 266.1 & $2.17 \mathrm{e}-03 \pm 1.7 \mathrm{e}-04$ & $0.24 \pm 0.07$ & $0.03 \pm 0.08$ & & \\
\hline RX J162335.8+780740 & 3 & 162335.89 & 7807 & 7740.0 & 17.3 & 12.7 & $2.54 \mathrm{e}-04 \pm 6.9 \mathrm{e}-05$ & $1.00 \pm 0.00$ & $0.20 \pm 0.26$ & U56725(19.3,-0.1,14.9) & -1.4 \\
\hline RX J162341.7+782114 & 4 & 162341.79 & 782 & 2114.2 & 21.7 & 19.0 & $6.96 \mathrm{e}-04 \pm 1.4 \mathrm{e}-04$ & $1.00 \pm 0.00$ & $1.00 \pm 0.00$ & U56892(16.0,0.6,10.5) & -2.5 \\
\hline RX J162420.6+775408 & 5 & 162420.63 & 775 & 5408.4 & 1.1 & 14673.9 & $4.31 \mathrm{e}-02 \pm 6.7 \mathrm{e}-04$ & $0.17 \pm 0.01$ & $0.06 \pm 0.02$ & U57802 $(14.8,1.9,4.3)$ & -1.7 \\
\hline RX J162441.2+774520 & 6 & 162441.29 & 774 & 4520.1 & 20.2 & 17.3 & $6.73 \mathrm{e}-04 \pm 1.4 \mathrm{e}-04$ & $1.00 \pm 0.00$ & $0.44 \pm 0.17$ & & \\
\hline RX J162457.6+780918 & 7 & 162457.66 & 780 & 9918.2 & 15.9 & 12.0 & $2.53 \mathrm{e}-04 \pm 6.9 \mathrm{e}-05$ & $1.00 \pm 0.00$ & $-0.09 \pm 0.31$ & U58672(16.6,0.6,14.1) & -2.7 \\
\hline RX J162459.0+780127 & 8 & 162459.05 & 780 & 0127.8 & 16.2 & 14.2 & $2.99 \mathrm{e}-04 \pm 7.4 \mathrm{e}-05$ & $1.00 \pm 0.00$ & $0.17 \pm 0.25$ & & \\
\hline RX J162500.2+781814 & 9 & 162500.29 & 7818 & 1815.0 & 17.8 & 14.3 & $3.35 \mathrm{e}-04 \pm 8.4 \mathrm{e}-05$ & $1.00 \pm 0.00$ & $0.14 \pm 0.27$ & & \\
\hline RX J162504.4+781152 & 10 & 162504.43 & 781 & 1152.6 & 10.0 & 46.2 & $6.37 \mathrm{e}-04 \pm 9.8 \mathrm{e}-05$ & $1.00 \pm 0.00$ & $0.21 \pm 0.18$ & & \\
\hline RX J162513.0+780300 & 11 & 162513.02 & 780 & 3300.0 & 11.2 & 19.5 & $2.92 \mathrm{e}-04 \pm 6.8 \mathrm{e}-05$ & $1.00 \pm 0.00$ & $0.16 \pm 0.22$ & U58965 $(18.2,0.6,12.6)$ & -2.0 \\
\hline RX J162515.4+775542 & 12 & 162515.49 & 775 & 5542.1 & 14.9 & 12.7 & $2.75 \mathrm{e}-04 \pm 7.2 \mathrm{e}-05$ & $1.00 \pm 0.00$ & $0.33 \pm 0.25$ & $\operatorname{NVSS}(9.2,1.9)$ & \\
\hline RX J162515.6+780113 & 13 & 162515.67 & 780 & 0113.6 & 13.6 & 13.6 & $2.37 \mathrm{e}-04 \pm 6.4 \mathrm{e}-05$ & $1.00 \pm 0.00$ & $-0.06 \pm 0.27$ & & \\
\hline RX J162517.0+775914 & 14 & $1625 \quad 17.10$ & 775 & 5914.7 & 13.0 & 16.4 & $3.09 \mathrm{e}-04 \pm 7.3 \mathrm{e}-05$ & $1.00 \pm 0.00$ & $0.73 \pm 0.22$ & U59114 $(19.2,0.3,22.2)$ & -1.5 \\
\hline RX J162531.3+775111 & 15 & 162531.32 & 775 & 5111.3 & 10.3 & 48.5 & $7.99 \mathrm{e}-04 \pm 1.2 \mathrm{e}-04$ & $0.97 \pm 0.27$ & $0.23 \pm 0.12$ & U59260 $(18.7,-0.3,8.8)$ & -1.0 \\
\hline RX J162534.4+774838 & 16 & 162534.43 & 7748 & 4838.5 & 18.8 & 13.8 & $3.98 \mathrm{e}-04 \pm 9.6 \mathrm{e}-05$ & $0.80 \pm 0.47$ & $0.42 \pm 0.17$ & & \\
\hline RX J162538.4+775002 & 17 & 162538.46 & 775 & 5002.7 & 14.0 & 33.0 & $6.77 \mathrm{e}-04 \pm 1.1 \mathrm{e}-04$ & $1.00 \pm 0.00$ & $0.35 \pm 0.12$ & U59471 $(17.9,-0.2,6.5)$ & -1.5 \\
\hline RX J162547.3+775816 & 18 & 162547.31 & 7758 & 5816.1 & 6.6 & 64.3 & $6.47 \mathrm{e}-04 \pm 9.1 \mathrm{e}-05$ & $0.30 \pm 0.17$ & $-0.17 \pm 0.13$ & U59661(13.8,0.8,5.7) & -3.5 \\
\hline RX J162547.6+781417 & 19 & 162547.60 & 781 & 1417.7 & 14.4 & 16.8 & $3.55 \mathrm{e}-04 \pm 8.2 \mathrm{e}-05$ & $1.00 \pm 0.00$ & $-0.39 \pm 0.24$ & & \\
\hline RX J162610.8+781045 & 20 & 162610.87 & 78 1 & 1045.7 & 4.5 & 140.6 & $1.05 \mathrm{e}-03 \pm 1.1 \mathrm{e}-04$ & $1.00 \pm 0.00$ & $0.16 \pm 0.10$ & & \\
\hline RX J162613.2+775730 & 21 & 162613.25 & 775 & 5730.2 & 13.8 & 10.5 & $2.25 \mathrm{e}-04 \pm 6.5 \mathrm{e}-05$ & $1.00 \pm 0.00$ & $0.12 \pm 0.26$ & U60332(16.9,0.4,10.1) & -2.6 \\
\hline RX J162624.7+781659 & 22 & 162624.77 & 7816 & 1659.7 & 4.6 & 327.7 & $2.86 \mathrm{e}-03 \pm 1.9 \mathrm{e}-04$ & $0.91 \pm 0.07$ & $0.33 \pm 0.06$ & F122_552 R20.3 :GClustr : $z=0.5$ & 0.1 \\
\hline RX J162636.9+775838 & 23 & 162636.99 & 7758 & 5838.5 & 10.8 & 18.4 & $2.94 \mathrm{e}-04 \pm 6.8 \mathrm{e}-05$ & $1.00 \pm 0.00$ & $0.03 \pm 0.21$ & & \\
\hline RX J162639.6+781944 & 24 & 162639.64 & $781 \mathrm{~s}$ & 1944.2 & 6.2 & 193.3 & $1.91 \mathrm{e}-03 \pm 1.6 \mathrm{e}-04$ & $0.64 \pm 0.09$ & $0.40 \pm 0.08$ & $\mathrm{U} 60881(17.9,-0.2,15.1)$ & -1.0 \\
\hline RX J162643.5+775757 & 25 & 162643.52 & 7757 & 5757.3 & 10.6 & 16.6 & $2.53 \mathrm{e}-04 \pm 6.3 \mathrm{e}-05$ & $1.00 \pm 0.00$ & $0.65 \pm 0.21$ & & \\
\hline RX J162646.7+780735 & 26 & 162646.80 & 780 & 7735.9 & 7.8 & 24.3 & $3.00 \mathrm{e}-04 \pm 6.4 \mathrm{e}-05$ & $1.00 \pm 0.00$ & $0.97 \pm 0.18$ & & \\
\hline RX J162703.2+775534 & 27 & 162703.27 & 775 & 5534.4 & 5.5 & 101.3 & $8.94 \mathrm{e}-04 \pm 1.1 \mathrm{e}-04$ & $0.70 \pm 0.16$ & $0.04 \pm 0.11$ & & \\
\hline RX J162703.7+782218 & 28 & 162703.75 & 782 & 2218.4 & 21.8 & 10.1 & $2.60 \mathrm{e}-04 \pm 7.6 \mathrm{e}-05$ & $1.00 \pm 0.00$ & $0.81 \pm 0.16$ & & \\
\hline RX J162718.9+775628 & 29 & $1627 \quad 18.91$ & 7756 & 5628.2 & 3.5 & 227.7 & $1.43 \mathrm{e}-03 \pm 1.2 \mathrm{e}-04$ & $0.67 \pm 0.10$ & $0.37 \pm 0.08$ & $\mathrm{U} 61755(17.9,2.1,4.6), \mathrm{WN}$ B1629.5+7802, $\operatorname{NVSS}(52.6,4.8)$ & -2.0 \\
\hline RX J162746.4+775411 & 30 & 162746.45 & 775 & 5411.3 & 5.3 & 89.4 & $8.02 \mathrm{e}-04 \pm 9.9 \mathrm{e}-05$ & $1.00 \pm 0.00$ & $0.38 \pm 0.11$ & & \\
\hline RX J162754.0+775130 & 31 & 162754.03 & 775 & 5130.5 & 12.3 & 15.5 & $3.00 \mathrm{e}-04 \pm 7.3 \mathrm{e}-05$ & $1.00 \pm 0.00$ & $0.21 \pm 0.18$ & & \\
\hline RX J162808.0+780437 & 32 & 162808.05 & 780 & 0437.2 & 5.6 & 84.4 & $7.66 \mathrm{e}-04 \pm 9.5 \mathrm{e}-05$ & $-0.20 \pm 0.09$ & $0.18 \pm 0.10$ & $\operatorname{NVSS}(4.8,3.2)$ & \\
\hline RX J162813.3+782100 & 33 & 162813.30 & 782 & 2100.2 & 10.3 & 57.7 & $7.94 \mathrm{e}-04 \pm 1.1 \mathrm{e}-04$ & $1.00 \pm 0.00$ & $0.42 \pm 0.14$ & & \\
\hline RX J162814.2+780336 & 34 & 162814.25 & 780 & 3336.2 & 1.1 & 3308.8 & $9.51 \mathrm{e}-03 \pm 3.0 \mathrm{e}-04$ & $0.35 \pm 0.03$ & $0.21 \pm 0.03$ & $\mathrm{U} 62860(17.6,-0.4,3.3), \mathrm{AGN} z=0.64$ (Y. Chu, priv. comm.) & -0.3 \\
\hline RX J162822.4+782221 & 35 & 162822.46 & 782 & 2221.8 & 11.4 & 56.8 & $8.18 \mathrm{e}-04 \pm 1.1 \mathrm{e}-04$ & $1.00 \pm 0.00$ & $0.59 \pm 0.12$ & & \\
\hline RX J162824.8+781033 & 36 & 162824.81 & 781 & 1033.9 & 7.7 & 26.9 & $3.12 \mathrm{e}-04 \pm 6.5 \mathrm{e}-05$ & $0.70 \pm 0.39$ & $-0.32 \pm 0.16$ & $\mathrm{U} 63068(14.8,1.5,7.9)$ & -3.7 \\
\hline RX J162826.5+780236 & 37 & 162826.55 & 7802 & 2236.4 & 12.6 & 13.7 & $2.07 \mathrm{e}-04 \pm 5.6 \mathrm{e}-05$ & $-0.82 \pm 0.05$ & $0.33 \pm 0.18$ & & \\
\hline RX J162834.8+780810 & 38 & 162834.84 & 7808 & 8810.4 & 11.0 & 11.1 & $1.78 \mathrm{e}-04 \pm 5.3 \mathrm{e}-05$ & $1.00 \pm 0.00$ & $-0.19 \pm 0.20$ & & \\
\hline RX J162835.4+781917 & 39 & 162835.50 & 781 1s & 1917.9 & 4.3 & 306.9 & $2.12 \mathrm{e}-03 \pm 1.6 \mathrm{e}-04$ & $0.41 \pm 0.07$ & $0.09 \pm 0.08$ & $\mathrm{U} 63298(18.3,0.0,5.0), \mathrm{F} 122 \_001$ AGN $z=1.134$ & -0.9 \\
\hline RX J162904.8+781030 & 40 & 162904.83 & 7810 & 1030.0 & 4.2 & 127.5 & $8.59 \mathrm{e}-04 \pm 9.7 \mathrm{e}-05$ & $0.58 \pm 0.14$ & $0.05 \pm 0.10$ & & \\
\hline RX J162910.4+780441 & 41 & 162910.47 & 780 & 0441.0 & 1.1 & 4240.2 & $1.23 \mathrm{e}-02 \pm 3.4 \mathrm{e}-04$ & $-0.99 \pm 0.00$ & $-0.12 \pm 0.03$ & U64063(13.3,0.0,2.3), RE J1629+780 WD binary & -2.1 \\
\hline RX J162913.3+780033 & 42 & 162913.34 & 780 & 0033.5 & 6.2 & 48.6 & $4.69 \mathrm{e}-04 \pm 7.6 \mathrm{e}-05$ & $0.65 \pm 0.26$ & $0.23 \pm 0.13$ & $\operatorname{APM}(20.7,0.7,4.6)$ & -0.8 \\
\hline RX J162923.9+781304 & 43 & 162923.90 & 781 & 1304.0 & 6.2 & 67.9 & $6.34 \mathrm{e}-04 \pm 8.8 \mathrm{e}-05$ & $0.71 \pm 0.20$ & $0.41 \pm 0.12$ & & \\
\hline RX J162928.5+780528 & 44 & 162928.57 & 780 & 5528.5 & 4.7 & 123.6 & $1.58 \mathrm{e}-03 \pm 1.3 \mathrm{e}-04$ & $-0.99 \pm 0.00$ & $0.07 \pm 0.07$ & & \\
\hline RX J162930.6+781545 & 45 & 162930.69 & 781 & 1545.5 & 8.3 & 47.2 & $5.14 \mathrm{e}-04 \pm 8.3 \mathrm{e}-05$ & $0 \pm 0.00$ & $0.19 \pm 0.15$ & & \\
\hline RX J162936.6+775814 & 46 & 162936.67 & 7758 & 5814.9 & 5.5 & 62.7 & $5.59 \mathrm{e}-04 \pm 8.2 \mathrm{e}-05$ & $0.51 \pm 0.19$ & $0.04 \pm 0.13$ & & \\
\hline RX J162938.0+775711 & 47 & 162938.06 & $775 ?$ & 5711.9 & 10.7 & 10.1 & $1.68 \mathrm{e}-04 \pm 5.1 \mathrm{e}-05$ & $1.00 \pm 0.00$ & $0.27 \pm 0.25$ & & \\
\hline RX J162945.0+781126 & 48 & 162945.04 & 781 & 1126.9 & 2.0 & 861.7 & $3.28 \mathrm{e}-03 \pm 1.8 \mathrm{e}-04$ & $0.28 \pm 0.05$ & $-0.06 \pm 0.05$ & U64758(17.7,2.0,1.0), F122_10 Star M5.5e & -1.7 \\
\hline RX J162956.3+781302 & 49 & 162956.39 & 781 & 1302.7 & 3.5 & 241.0 & $1.31 \mathrm{e}-03 \pm 1.2 \mathrm{e}-04$ & $0.66 \pm 0.10$ & $0.17 \pm 0.09$ & & \\
\hline RX J162956.5+781705 & 50 & 162956.51 & 7817 & 1705.3 & 12.2 & 16.9 & $2.68 \mathrm{e}-04 \pm 6.5 \mathrm{e}-05$ & $1.00 \pm 0.00$ & $-0.02 \pm 0.25$ & U65068 $(19.4,0.6,6.5)$ & -1.6 \\
\hline
\end{tabular}


W. Pietsch and H. Arp: A possible X-ray jet from the starburst galaxy NGC 6217

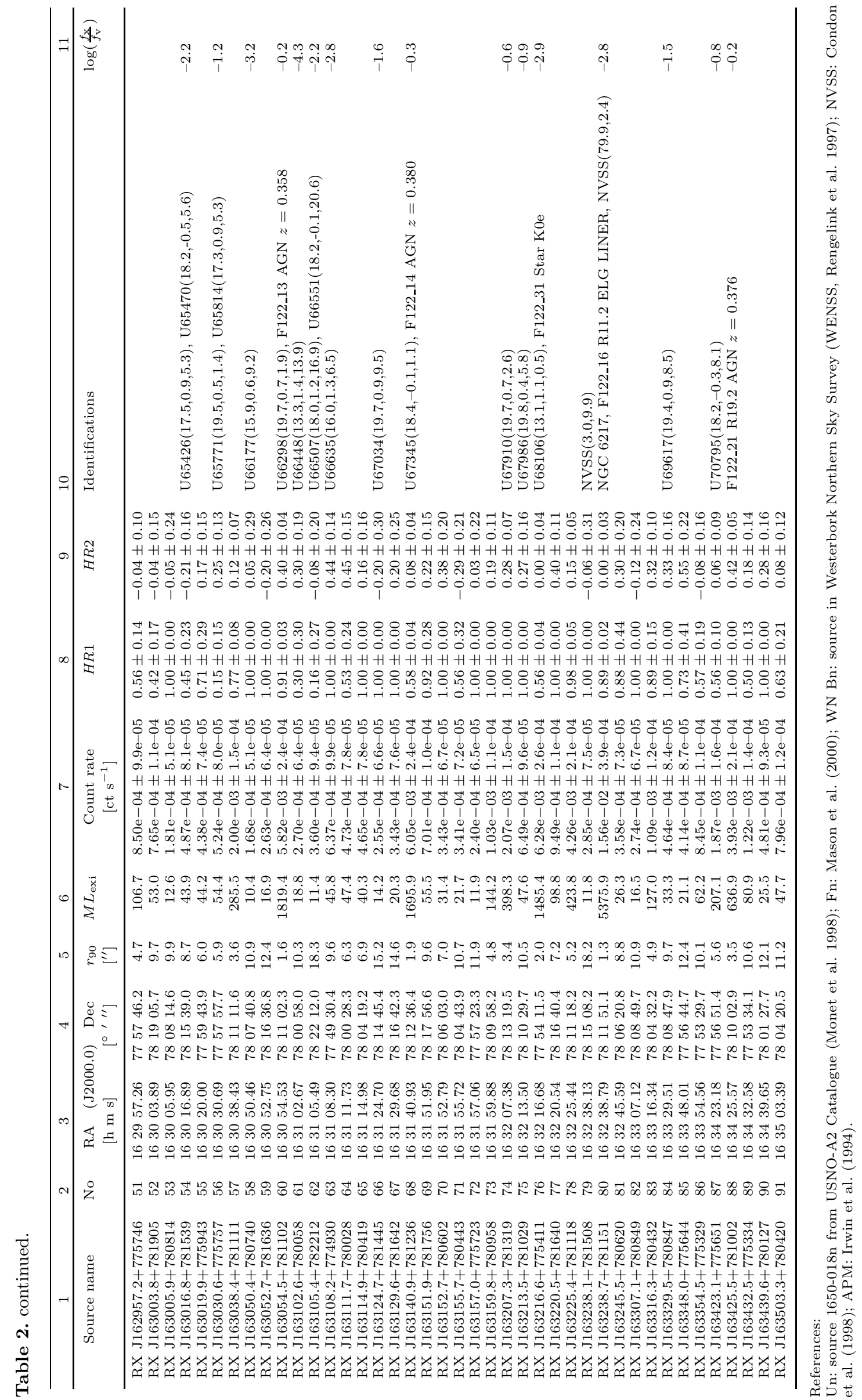




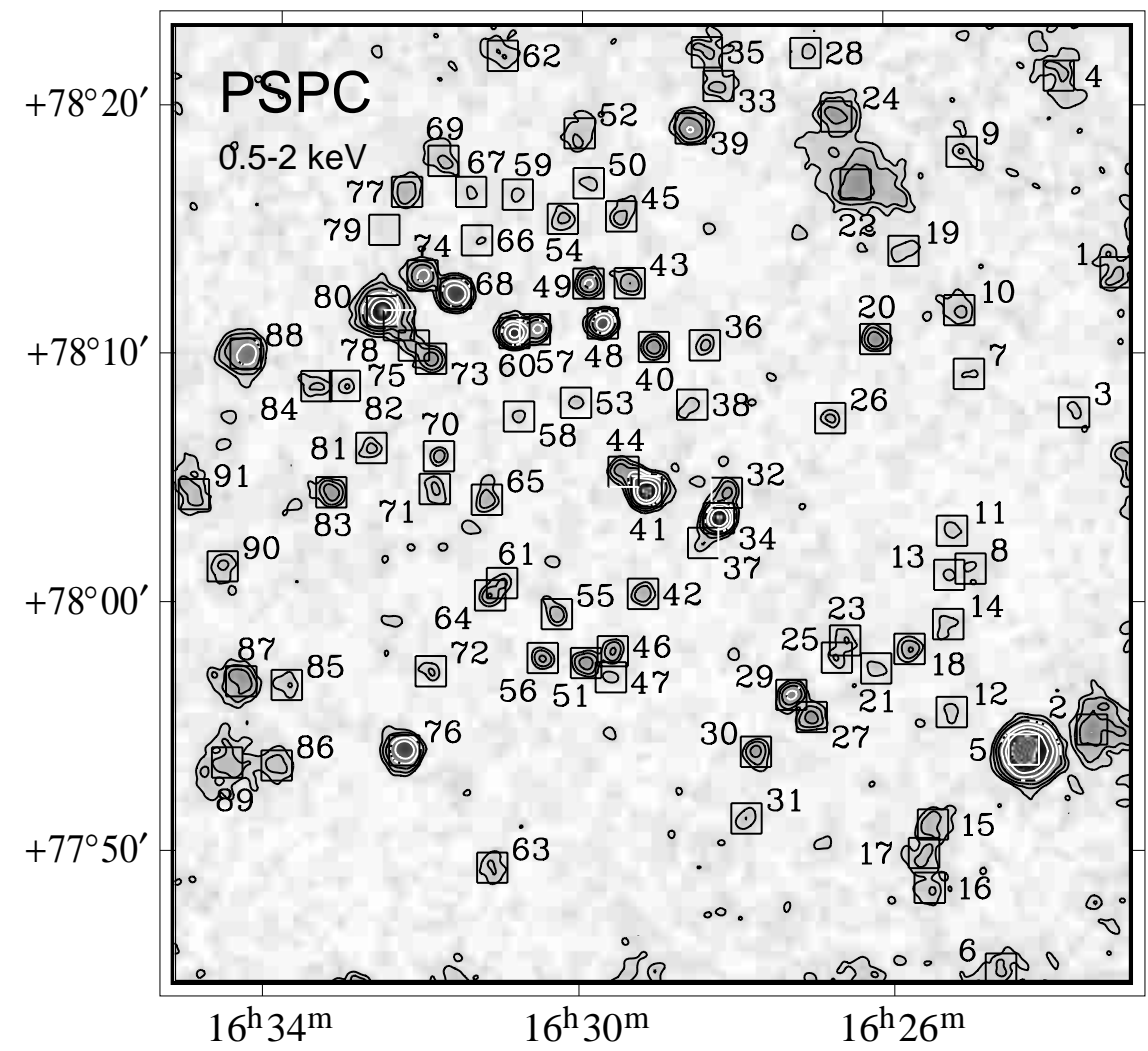

Fig. A.1. Contour plot superimposed on exposure corrected and smoothed gray-scale image of the ROSAT PSPC hard band (0.5-2 keV, $117 \mathrm{ks}$ low background integration time) of the $38.3 \times$ 38 '. 3 field centered on RE J1629+780. Contour levels are the same as in Fig. 1. Sources from the catalog are given as boxes with source numbers

\section{Appendix A: Identification of $X$-ray sources detected in the field}

Ninety-one sources are detected in the $38^{\prime} .3 \times 38^{\prime} \cdot 3$ field centered on RE J1629+780 in the deep PSPC exposure. Table 2 shows their characteristics. Sources 5, 22, 34, 41, $44,57,68,78$, and 80 have been flagged as extended by the detection algorithm. While for most of them this might indicate overlapping PSF of nearby point sources, extended emission in 22 further confirms the galaxy cluster identification of the RIXOS program. Extended emission in 80 reflects overlapping emission components from the hot interstellar medium and bright point sources in the NGC 6217 . The cases of source 5 and $41 / 44$ will be discussed separately.

To guide optical identifications we produced overlay plots of PSPC $0.5-2.0 \mathrm{keV}$ band contours on the DSS2 red and blue plates and inspected APM (Irwin et al. 1994) finding charts and the USNO-A2 Catalogue (Monet et al. 1998) from which we get positional accuracies for the optical candidates of better than $1^{\prime \prime}$ for USNO-A2 (2" for APM), optical magnitudes of the blue $(\mathrm{O})$ and red (E) plates, and the color index O-E. Optical counterparts were searched for in the $\sim r_{90} \mathrm{X}$-ray error circle. Usually only one optical source correlates if at all. For 33 sources we give the higher accuracy USNO-A2 identification candidate information in Col. 10 of Table 2 (source number with magnitude from blue plate, the color index, and separation in arcsec between optical and X-ray position following in parenthesis). For source 42 only APM information is available. For sources 54, 56, and 62 two USNO-A2 sources are candidates for the identification.

The bright source 5 includes 3 USNO-A2 sources within its extent. The best correlation is given in the table. There are, however, two fainter objects within $15^{\prime \prime}$, i.e. U57709 $(18.2,0.7,12.6)$ and U57766 $(16.7,1.5,14.7)$. Comparing POSS I and II plates we find that the best correlating optical source U57802 is a newly detected fast moving object with a proper motion $\left(-170{\text { mas } \mathrm{yr}^{-1}}^{-1}\right.$ in $\mathrm{RA} \times \cos (\mathrm{Dec})$ and 110 mas yr$^{-1}$ in Dec). Most certainly two or even all three optical candidates contribute to the X-ray emission.

To investigate the reliability of the identifications and allow rough classification in foreground stars and AGN, we have added in Col. $11 \log \left(f_{\mathrm{x}} / f_{\mathrm{v}}\right)=\log \left(f_{\mathrm{x}}\right)+\left(m_{\mathrm{v}} / 2.5\right)+$ 5.37 (see Maccacaro et al. 1988). This X-ray to optical luminosity relation can be used to discriminate between stars, for which $\log \left(f_{\mathrm{x}} / f_{\mathrm{v}}\right)$ is generally $<-1$, and AGN for which this quantity is in the range -1.2 to +1.2 (see also Pietsch et al. 1998; Vogler \& Pietsch 1999). X-ray fluxes in the $0.5-2.0 \mathrm{keV}$ band have been determined from the count rates assuming a power law spectrum with photon index of 2, corrected for Galactic absorption (see Sect. 2). For $f_{\mathrm{v}}$ we used the red magnitude of the first identification candidate.

Some of the brighter X-ray sources in Fig. 3 (sources 22, 39, 48, 68, 76, 80, and 88) have already been investigated in the RIXOS program (Nicholson et al. 1997; Puchnarewicz et al. 1997; Mason et al. 2000, see Col. 10 of Table 2). They were identified with the help of optical 
spectroscopy as a cluster of galaxies, AGN, star, AGN, AGN, star, emission line galaxy, and AGN, respectively. The AGN and stars nicely confirm the $\log \left(f_{\mathrm{x}} / f_{\mathrm{v}}\right)$ classification scheme. One of the AGN (source 60 with a redshift of 0.358 ) is very red and could be absorbed by some outlying dust from NGC 6217 . This would be a further incentive to spectroscopically identify the fainter candidates near the galaxy.

While in the soft band the emission of RE J1629+780 dominates the central ares of the field, in the $0.5-2.0 \mathrm{keV}$ band there is a smooth transition from source 41 (i.e. RE J1629+780) to 44 indicating the contribution from even another source at intermediate position which from the optical overlays can be identified with a blue stellar object cataloged in APM with a blue magnitude of 20.1, color 0.1, at position (epoch J2000.0) RA $=16^{\mathrm{h}} 29^{\mathrm{m}} 18^{\mathrm{s}} .9$, Dec $=+78^{\circ} 05^{\prime} 14^{\prime \prime}$.

We find five correlations of the X-ray source list with the $1.4 \mathrm{GHz}$ NRAO/VLA Sky Survey (NVSS, Condon et al. 1998) radio source catalogs, one of which is also contained in the $325 \mathrm{MHz}$ Westerbork Northern Sky Survey (WENSS, Rengelink et al. 1997) catalog. The brightest source is NGC 6217. The second brightest source is also identified with a optical source and most likely is, based on the optical color and $\log \left(f_{\mathrm{x}} / f_{\mathrm{v}}\right)$, a radio galaxy. The other three sources have no optical counterparts and are most likely radio-loud AGN.

\section{References}

Arp, H. 1966a, ApJS, 14, 1

Arp, H. 1966b, Atlas of peculiar galaxies (Pasadena: California Inst. Technology)

Aschenbach, B. 1988, Appl. Opt., 27, 1404

Böhringer, H., Belsole, E., Kennea, J., et al. 2001, A\&A, 365, L181

Bonatto, C., Pastoriza, M. G., Alloin, D., \& Bica, E. 1998, A\&A, 334, 439

Cecil, G., Greenhill, L. J., DePree, C. G., et al. 2000, ApJ, 536, 675

Condon, J. J. 1987, ApJS, 65, 485

Condon, J. J., Cotton, W. D., Greisen, E. W., et al. 1998, AJ, 115,1693

Condon, J. J., Helou, G., Sanders, D. B., \& Soifer, B. T. 1990, ApJS, 73, 359

Cooke, B. A., Barstow, M. A., Pye, J. P., et al. 1992, Nature, 355,61

Dahlem, M., Weaver, K. A., \& Heckman, T. M. 1998, ApJS, 118,401

Dickey, J. M., \& Lockman, F. J. 1990, ARA\&A, 28, 215

Döbereiner, S., Junkes, N., Wagner, S. J., et al. 1996, ApJ, 470, L15
Elfhag, T., Booth, R. S., Hoeglund, B., Johansson, L. E. B., \& Sandqvist, A. 1996, A\&AS, 115, 439

Hasinger, G., Burg, R., Giacconi, R., et al. 1998, A\&A, 329, 482

Hummel, E., van der Hulst, J. M., \& Dickey, J. M. 1984, A\&A, 134,207

Irwin, M., Maddox, S., \& McMahon, R. 1994, Spectrum, 2, 14

Kellermann, K. I., Sramek, R., Schmidt, M., Shaffer, D. B., \& Green, R. 1989, AJ, 98, 1195

Kennicutt, R. C. 1992, ApJ, 388, 310

Kraft, R. P., Forman, W., Jones, C., et al. 2000, ApJ, 531, L9

Maccacaro, T., Gioia, I. M., Wolter, A., Zamorani, G., \& Stocke, J. T. 1988, ApJ, 326, 680

Malkan, M. A., Gorjian, V., \& Tam, R. 1998, ApJS, 117, 25

Marshall, H. L., Harris, D. E., Grimes, J. P., et al. 2001, ApJ, 549, L167

Mason, K. O., Carrera, F. J., Hasinger, G., et al. 2000, MNRAS, 311, 456

Mittaz, J. P. D., Carrera, F. J., Romero-Colmenero, E., et al. 1999, MNRAS, 308, 233

Monet, D., Bird, A., Canzian, B., Dahn, C., et al. 1998, A catalogue of astrometric standards

Moran, E. C., Kay, L. E., Davis, M., Filippenko, A. V., \& Barth, A. J. 2001, ApJ, 556, 75

Nicholson, K. L., Mittaz, J. P. D., \& Mason, K. O. 1997, MNRAS, 285, 831

Pfeffermann, E., Briel, U. G., Hippmann, H., et al. 1987, Proc. SPIE, 733, 519

Pietsch, W., Trinchieri, G., \& Vogler, A. 1998, A\&A, 340, 351

Pietsch, W., Vogler, A., Kahabka, P., Jain, A., \& Klein, U. 1994, A\&A, 284, 386

Pietsch, W., Vogler, A., Klein, U., \& Zinnecker, H. 2000, A\&A, 360,24

Polletta, M., Bassani, L., Malaguti, G., Palumbo, G. G. C., \& Caroli, E. 1996, ApJS, 106, 399

Puchnarewicz, E. M., Mason, K. O., Carrera, F. J., et al. 1997, MNRAS, 291, 177

Rengelink, R. B., Tang, Y., de Bruyn, A. G., et al. 1997, A\&AS, 124,259

Sandage, A., \& Tammann, G. A. 1981, A revised ShapleyAmes Catalog of bright galaxies (Washington: Carnegie Institution)

Schwartz, D. A., Marshall, H. L., Lovell, J. E. J., et al. 2000, ApJ, 540, L69

Trümper, J. 1982, Adv. Space Res., 2(4), 241

Veron-Cetty, M.-P. \& Veron, P. 1998, A Catalogue of quasars and active nuclei, 8th ed. (Garching: ESO Scientific Report Ser. vol. 18)

Vogler, A., \& Pietsch, W. 1999, A\&A, 342, 101

Wilson, A. S., Young, A. J., \& Shopbell, P. L. 2001, ApJ, 547, 740

Zimmermann, H. U., Becker, W., Belloni, T., et al. 1998, EXSAS User's Guide, 4th ed. (Garching: MPE Report 257) 